\title{
Modified Adaptive Control for Region 3 Operation in the Presence of Wind Turbine Structural Modes
}

\author{
Susan A. Frost ${ }^{*}$ \\ NASA Ames Research Center, Moffett Field, CA, 94035 \\ Mark J. Balas ${ }^{\dagger}$ \\ University of Wyoming, Laramie, WY, 82071 \\ and \\ Alan D. Wright ${ }^{\ddagger}$ \\ National Renewable Energy Laboratory, Golden, CO, 80401
}

\begin{abstract}
Many challenges exist for the operation of wind turbines in an efficient manner that is reliable and avoids component fatigue and failure. Turbines operate in highly turbulent environments resulting in aerodynamic loads that can easily excite turbine structural modes, possibly causing component fatigue and failure. Wind turbine manufacturers are highly motivated to reduce component fatigue and failure that can lead to loss of revenue due to turbine down time and maintenance costs. The trend in wind turbine design is toward larger, more flexible turbines that are ideally suited to adaptive control methods due to the complexity and expense required to create accurate models of their dynamic characteristics. In this paper, we design an adaptive collective pitch controller for a high-fidelity simulation of a utility-scale, variable-speed horizontal axis wind turbine operating in Region 3 . The objective of the adaptive pitch controller is to regulate generator speed, accommodate wind gusts, and reduce the excitation of structural modes in the wind turbine. The control objective is accomplished by collectively pitching the turbine blades. The adaptive collective pitch controller for Region 3 was compared in simulations with a baseline classical Proportional Integrator (PI) collective pitch controller. The adaptive controller will demonstrate the ability to regulate generator speed in Region 3, while accommodating gusts, and reducing the excitation of certain structural modes in the wind turbine.
\end{abstract}

\section{Introduction}

$\mathrm{R}$ ATED wind speed is the velocity at which maximum power output, or rated power, of a wind turbine is achieved. If a turbine is allowed to operate in an uncontrolled manner, in conditions where the wind speed is above the rated wind speed, the power output would increase in proportion to the cube of the wind speed, resulting in overheating of the generator and the power electronics system. Additionally, high wind speeds result in larger aerodynamic forces on the machine, possibly leading to system fatigue and failure. Hence, power output of a turbine must be held constant for wind speeds at, and above, the turbine's rated wind speed. This operation region is referred to as Region $3^{1}$.

Turbine power output should be maintained at rated power when operating in Region 3. For variable-speed turbines, a constant torque is applied at the generator, and the turbine rotational speed is maintained at the desired value through the use of blade pitch. In some machines, the pitch angle of each blade is adjusted identically (collective blade pitch); in others the blade pitch is adjusted independently of the other blades (independent blade

\footnotetext{
${ }^{*}$ Research Engineer, Intelligent Systems Division, M/S 269-2, AIAA Member.

${ }^{\dagger}$ Professor \& Dept. Head, Department of Electrical and Computer Engineering, 1000 E. University Avenue, AIAA Fellow.

${ }^{\ddagger}$ Senior Engineer II, National Renewable Energy Laboratory, M/S 381 1, 1617 Cole Blvd., AIAA Member. 
pitch). Collective blade pitch control is a well-accepted approach to regulating turbine speed and responding to changes in wind speed ${ }^{2}$.

Wind turbine control problems can benefit from adaptive control techniques ${ }^{3,4}$, which are well suited to nonlinear applications that have unknown modeling parameters and poorly known operating conditions. The main nonlinearities in a wind turbine model come from the nonlinear aerodynamic loads on the turbine. Creating an accurate model of the dynamic characteristics of a wind turbine is expensive and extremely difficult, if not impossible. Additionally, wind turbines operate in highly turbulent and unpredictable conditions. These complex aspects of wind turbines make them attractive candidates for the application of adaptive control methods. In this paper, we focus on the direct adaptive control (DAC) approach developed in refs. 5-6. This approach has been extended to handle adaptive rejection of persistent disturbances ${ }^{7,8}$.

The literature suggests that direct adaptive control methods have rarely been used on utility-scale horizontal axis wind turbines (HAWTs). It was shown in Ref. 9 that a pitch controller designed with Direct Model Reference Adaptive Control (DMRAC) was comparable to a PID pitch controller when regulating turbine speed in a simulation of a rigid, nonlinear model of a HAWT. Adaptive pitch control to optimize power in Region 2 of the Controls Advanced Research Turbine (CART) was demonstrated to be effective in simulations and field tests ${ }^{10}$. The CART is a utility-scale, variable-speed HAWT.

In this paper, a direct adaptive control approach is used to design an adaptive collective pitch controller to operate in Region 3 in conjunction with the Fatigue, Aerodynamics, Structures, and Turbulence Codes (FAST), a high-fidelity simulation of the CART ${ }^{1}$. The objective of the adaptive pitch controller is to regulate generator speed and to reject step disturbances in the presence of turbine structural modes. This objective is accomplished by collectively pitching the turbine blades. It has been demonstrated that the uniform wind disturbance, without shear, across the rotor disk of a turbine can be accurately accounted for when modeled as a step disturbance of unknown amplitude ${ }^{11,12}$. We designed the adaptive collective pitch controller to reject step disturbances to improve controller performance.

As wind turbines become larger and more flexible, the turbine structural modes can be more easily excited by wind gusts and the actions of the controller. For this reason, the adaptive Region 3 collective pitch controller was augmented to compensate for structural modes of the turbine that might be excited by aerodynamic loads or the control input. The adaptive pitch controller was implemented in the FAST simulation of the CART and tested with step wind inflow and turbulent wind inflow. Simulations were run with various wind turbine flexibility modes enabled. Comparisons of the generator speed errors were made between the simulation results of the adaptive pitch controller and a baseline Proportional Integrator (PI) pitch controller.

\section{CART Configuration and FAST Simulator Specifications}

The CART is a two-bladed, upwind, active-yaw, variable-speed HAWT located at the National Wind Technology Center (NWTC) in Golden, Colorado. This machine is used as a test bed to study aspects of wind turbine control technology for medium-scale machines ${ }^{1}$. The rated generator speed for the CART is $1800 \mathrm{rpm}$.

The CART has been modeled with the FAST Codes as a combination of rigid and flexible bodies connected by several degrees of freedom (DOFs). The FAST Code is a comprehensive aeroelastic simulator capable of predicting both the extreme loads and the fatigue loads of two- and three-bladed horizontal axis wind turbines ${ }^{13}$. Results from FAST simulations of the CART compared favorably with field tests of similar controllers on the actual CART ${ }^{11}$.

\section{Direct Adaptive Control with Rejection of Persistent Disturbances}

In this section, we describe the formulation of a direct adaptive control approach with adaptive rejection of persistent disturbances ${ }^{8}$. The plant is assumed to be well modeled by the linear, time-invariant, finite-dimensional system given by:

$$
\left\{\begin{array}{l}
\dot{x}_{p}=A_{p} x_{p}+B_{p} u_{p}+\Gamma_{p} u_{D} \\
y_{p}=C_{p} x_{p} ; \quad x_{p}(0)=x_{0}
\end{array}\right.
$$

where the plant state, $x_{p}$ is an $\mathrm{N}_{\mathrm{p}}$-dimensional vector, the control input vector, $u_{p}$, is M-dimensional, and the sensor output vector, $y_{p}$, is $\mathrm{P}$-dimensional. The disturbance input vector, $u_{D}$, is $\mathrm{M}_{\mathrm{D}}$-dimensional and will be thought to come from the Disturbance Generator: 


$$
\left\{\begin{array}{l}
u_{D}=\Theta z_{D} \\
\dot{z}_{D}=F z_{D} ; \quad z_{D}(0)=z_{0}
\end{array}\right.
$$

where the disturbance state, $z_{D}$, is $\mathrm{N}_{\mathrm{D}}$-dimensional. All matrices in Eqs. (1)-(2) have the appropriate compatible dimensions. Such descriptions of persistent disturbances were first used in Ref. 14 to describe signals of known form but unknown amplitude. Equation (2) can be rewritten in a form that is not a dynamical system, which is sometimes easier to use:

$$
\left\{\begin{array}{l}
u_{D}=\Theta z_{D} \\
z_{D}=L \phi_{D}
\end{array}\right.
$$

where $\phi_{D}$ is a vector composed of the known basis functions for the solution of $u_{D}=\Theta z_{D}$, i.e., $\phi_{D}$ are the basis functions which make up the known form of the disturbance, and $L$ is a matrix of dimension $N_{D}$ by $\operatorname{dim}\left(\phi_{D}\right)$. The method for rejecting persistent disturbances used in this paper requires only the knowledge of the form of the disturbance. The amplitude of the disturbance does not need to be known, i.e. $(L, \Theta)$ can be unknown. In this paper, we will be interested in rejecting step disturbances of unknown amplitude which can be represented in the form of Eq. (3) as $\phi_{D} \equiv 1$, with $(L, \Theta)$ unknown.

In Ref. 7, as with much of the control literature, it is assumed that the plant and disturbance generator parameter matrices $(A, B, C, \Gamma, \Theta, F)$ are known. This knowledge of the plant and its disturbance generator allows the Separation Principle of Linear Control Theory to be invoked to arrive at a State-Estimator based, linear controller that can suppress the persistent disturbances via feedback. In this paper, we will not assume that the plant and disturbance generator parameter matrices $(A, B, C, \Gamma, \Theta)$ are known. But, we will assume that we know the disturbance generator parameter, $F$, from Eq. (2), i.e., the form of the disturbance functions is known. In many cases, knowledge of $F$ is not a severe restriction, since the disturbance function is often of known form but unknown amplitude.

The control objective will be to cause the output of the plant, $y_{p}$, to asymptotically track zero while accommodating disturbances of the form given by the disturbance generator. We define the output error vector as:

$$
e_{y} \equiv y_{p}-0
$$

To achieve the desired control objective, we want:

$$
e_{y} \underset{t \rightarrow \infty}{\longrightarrow} 0 \text {. }
$$

Consider the plant given by Eq. (1) with the disturbance generator given by Eq. (3). The control objective for this system will be accomplished by an adaptive control law of the form:

$$
u_{p}=G_{e} e_{y}+G_{D} \phi_{D}
$$

where $G_{e}$ and $G_{D}$ are matrices of the appropriate compatible dimensions, whose definitions will be given later. In Ref. 8, we developed the gain adaptation laws to make asymptotic output regulation possible. The adaptive gain laws that produce asymptotic tracking are:

$$
\left\{\begin{array}{l}
\dot{G}_{e}=-e_{y} e_{y}^{T} h_{e} \\
\dot{G}_{D}=-e_{y} \phi_{D}^{T} h_{D}
\end{array}\right.
$$

where $h_{11}$ and $h_{22}$ are arbitrary, positive definite matrices. In ref. 8, we analyzed the stability of this controller and showed that the adaptive gains, $G_{e}$ and $G_{D}$, remain bounded and asymptotic tracking occurs, i.e., $e_{y} \underset{t \rightarrow \infty}{\longrightarrow} 0$ 
when the system $(A, B, C)$ is almost strict positive real (ASPR). Recall that ASPR means $C B>0$ and $P(s)=C(s I-A)^{-1} B$ is minimum phase.

\section{Adaptive Collective Pitch Controller}

In this section, we describe the adaptive collective pitch controller that was designed to regulate generator speed and reject step disturbances. More details on the design of this controller and its simulation results can be found in Ref. 15. The plant used in the FAST simulation of the CART is a two bladed wind turbine model with turbine aerodynamics and generator degree of freedom enabled. The plant neglected all other dynamics and degrees of freedom. The FAST Codes were used to model the nonlinear aerodynamic loads on the turbine in our plant. A state space model of the plant was generated by the FAST Codes. See Refs. 13 and 16 to obtain the model. The output of the plant was the generator speed. The control input was the collective blade pitch angle command.

A classical PI collective pitch controller (the baseline PI pitch controller) has been implemented and tested in the FAST simulation of the CART ${ }^{2}$. A similar version of the baseline PI pitch controller has been tested on the actual CART $^{11}$. The baseline PI pitch controller in the FAST simulator provides a basis for comparison with the adaptive pitch controller. The control goal of a collective pitch controller for a wind turbine operating in Region 3 is to regulate generator speed while accommodating wind gusts. This goal is accomplished by collectively pitching the turbine blades. The FAST simulator model of the CART measures generator speed and feeds it back to the baseline PI pitch controller which regulates it to the rated generator speed for the wind turbine.

The adaptive collective pitch controller designed for this paper replaces the baseline PI pitch controller in the FAST simulator with the same input and output. The adaptive pitch controller is designed with the direct adaptive control approach described in Section III. The control objective is generator speed regulation and rejection of persistent step disturbances. The step disturbances account for wind speed fluctuations and wind gusts that the turbine may experience during operation. The control objective is accomplished by an adaptive control law of the form given in Eq. (6) with gains specified in Eq. (7).

For a step function, the disturbance generator function in the form of Eq. (3) is specified by $\phi_{D}=1$. Recall that we are not required to know the amplitude of the disturbance function, i.e., $(L, \Theta)$ from Eq. (3) need not be known. The adaptive control law that accomplishes the control goals described above is:

$$
\left\{\begin{array}{l}
u_{p}=G_{e} e_{y}+G_{D} \\
\dot{G}_{e}=-e_{y} e_{y}^{T} h_{e} \\
\dot{G}_{D}=-e_{y} h_{D}
\end{array}\right.
$$

where $h_{e}, h_{D}>0$.

The adaptive controller specified by Eq. (8) was implemented in Simulink in the FAST simulation of the CART. The adaptive controller gains, $h_{e}$ and $h_{D}$, were tuned to minimize the generator speed error, since we had the goal of regulating generator speed, while keeping the blade pitch rate in a range similar to that of the baseline PI controller. The values of the gains used in the adaptive controller were: $h_{e}=4.0$ and $h_{D}=0.3$.

The adaptive controller was tested using the FAST simulator of the CART. The simulations were run from time 0 seconds to 100 seconds with an integration step size of 0.006 seconds. The generator DOF switch was turned on. During the initial tests, the other DOF switches were turned off. The wind turbine had fixed-yaw with no yaw control. Aerodynamic forces were calculated during the runs. The parametric information for the FAST simulator as we configured it is available from Refs. 13 and 16.

Step wind inflow, resulting in Region 3 operation, was used for the FAST simulations, see Fig. 1. Generator speed errors for the baseline PI pitch controller and the adaptive pitch controller are shown in Fig. 2. All controller performance figures start at time equals 20 seconds, allowing transients due to system startup to die off. The adaptive controller demonstrated robust performance with smaller tracking errors than the baseline controller ${ }^{15}$.

We subsequently observed that when the drive train rotational DOF and the first blade flap DOF were enabled in the FAST simulation, both the baseline PI controller and the adaptive controller had difficulties regulating generator speed with collective blade pitch, see Fig. 3. This observation led us to investigate the excitation of certain structural modes of the turbine during operation. In particular, we focused on the excitation of the first drive train torsional mode, which is generally one of the lower frequency modes in a wind turbine. 
The FAST simulation of the CART allows for the measurement of many system parameters. We measured the low-speed shaft torque and observed that there was a $3.47 \mathrm{~Hz}$ disturbance in the measurements. This is consistent with the first drive train torsional mode that is estimated at $3.5 \mathrm{~Hz}$. A low-pass filter was designed to filter out this mode in the generator speed that is fed back to the controller. The transfer function for the low-pass filter is given by $F(s)=\frac{10}{s+10}$. The results of incorporating the low-pass filter with the baseline PI controller are shown in Fig. 4. The low-pass filter improves the regulation of the generator speed for the PI controller, but the controller still has difficulties, especially for higher wind speeds. In the next section, we introduce more rigorous techniques to reduce the effects of structural mode through the use of a residual mode filter.

\section{Residual Mode Filter Augmentation of Adaptive Controller}

In some cases the plant in Eq. (1) does not satisfy the requirements of ASPR. Instead, there maybe be a modal subsystem that inhibits this property. This section will present new results for our adaptive control theory developed in Ref. 7-8. We will modify the adaptive controller with a Residual Mode Filter (RMF) to compensate for the troublesome modal subsystem, as was done in Ref. 18 for fixed gain non-adaptive controllers. Here we present new theory for adaptive controllers modified by Residual Mode Filters.

Let us assume that Eq. (1) can be partitioned into the following form:

$$
\left\{\begin{array}{l}
{\left[\begin{array}{c}
\dot{x} \\
\dot{x}_{Q}
\end{array}\right]=\left[\begin{array}{cc}
A & 0 \\
0 & A_{Q}
\end{array}\right]\left[\begin{array}{c}
x \\
x_{Q}
\end{array}\right]+\left[\begin{array}{c}
B \\
B_{Q}
\end{array}\right] u_{p}+\left[\begin{array}{l}
\Gamma \\
0
\end{array}\right] u_{D}} \\
y_{p}=\left[\begin{array}{ll}
C & C_{Q}
\end{array}\right]\left[\begin{array}{c}
x \\
x_{Q}
\end{array}\right]
\end{array}\right.
$$

with $x_{p} \equiv\left[\begin{array}{c}x \\ x_{Q}\end{array}\right], A_{p}=\left[\begin{array}{cc}A & 0 \\ 0 & A_{Q}\end{array}\right], B_{p}=\left[\begin{array}{c}B \\ B_{Q}\end{array}\right], \Gamma_{p}=\left[\begin{array}{l}\Gamma \\ 0\end{array}\right], C_{p}=\left[\begin{array}{ll}C & C_{Q}\end{array}\right]$, and Disturbance Generator $\left\{\begin{array}{l}\dot{z}_{D}=F z_{D} \\ u_{D}=\theta z_{D}\end{array}\right.$ or $z_{D}=L \phi_{D}$ as given in Eqs. (2)-(3).

The Output Tracking Error remains as in Eq. (4) and our control objective is as in Eq. (5), i.e. $e_{y} \equiv y_{p} \underset{t \rightarrow \infty}{\longrightarrow} 0$. However, now we will only assume that the subsystem $(A, B, C)$ is Almost Strictly Positive Real (ASPR), rather than the full un-partitioned plant $\left(A_{p}=\left[\begin{array}{cc}A & 0 \\ 0 & A_{Q}\end{array}\right], B_{p}=\left[\begin{array}{c}B \\ B_{Q}\end{array}\right], C_{p}=\left[\begin{array}{ll}C & C_{Q}\end{array}\right]\right)$, and the modal subsystem $\left(A_{Q}, B_{Q}, C_{Q}\right)$ will be known and open-loop stable, i.e. $A_{Q}$ is stable. Also note that this subsystem is considered to be unaffected by the disturbance input. This latter assumption may not be perfectly true, but will be a reasonable approximation of the plant behavior. So, in summary, the actual plant has an ASPR subsystem and a known modal subsystem that is stable but inhibits the property of ASPR for the full plant. Hence, this modal subsystem must be compensated or filtered away.

We define the Residual Mode Filter (RMF):

$$
\left\{\begin{array}{l}
\dot{\hat{x}}_{Q}=A_{Q} \hat{x}_{Q}+B_{Q} u_{p} \\
\hat{y}_{Q}=C_{Q} \hat{x}_{Q}
\end{array}\right.
$$

And the compensated tracking error:

$$
\tilde{e}_{Q} \equiv y_{p}-\hat{y}_{Q}
$$

Now we let $e_{Q} \equiv \hat{x}_{Q}-x_{Q}$ and obtain: 


$$
\dot{e}_{Q}=A_{Q} e_{Q}
$$

Consequently, we have:

$$
\begin{aligned}
\tilde{e}_{y} & \equiv y_{p}-\hat{y}_{Q}=C x+C_{Q} x_{Q}-\left[C_{Q} x_{Q}+C_{Q} e_{Q}\right] \\
& =C x-C_{Q} e_{Q}
\end{aligned}
$$

As in Ref. 7-8, we define the Ideal Trajectories, but only for the ASPR Subsystem:

$$
\left\{\begin{array}{l}
\dot{x}_{*}=A x_{*}+B u_{*}+\Gamma u_{D} \\
y_{*}=C x_{*}=0
\end{array}\right.
$$

with $\left\{\begin{array}{l}x_{*}=S_{1}^{*} z_{D} \\ u_{*}=S_{2}^{*} z_{D}\end{array}\right.$. This is equivalent to the Matching Conditions:

$$
\left\{\begin{array}{l}
S_{1}^{*} F=A S_{1}^{*}+B S_{2}^{*}+\Gamma \theta \\
C S_{1}^{*}=0
\end{array}\right.
$$

which are known to be uniquely solvable when $C B$ is nonsingular. However, we do not need to know the actual solutions for this adaptive control approach to be applied.

$$
\begin{aligned}
& \text { Let }\left\{\begin{array}{l}
\Delta x \equiv x-x_{*} \\
\Delta u \equiv u_{p}-u_{*} \\
\Delta \tilde{y} \equiv \tilde{e}_{y}=C x-C_{Q} e_{Q}
\end{array}\right. \text {. Then we can write: } \\
& \left\{\begin{array}{l}
\Delta \dot{x}=A \Delta x+B \Delta u \\
\Delta \tilde{y}=C x-C_{Q} e_{Q}=C x-y_{*}-C_{Q} e_{Q}=C \Delta x-C_{Q} e_{Q}
\end{array}\right.
\end{aligned}
$$

because, from Eq. (14), $y_{*}=0$. This system can be rewritten as:

$$
\left\{\begin{array}{l}
{\left[\begin{array}{c}
\Delta \dot{x} \\
\dot{e}_{Q}
\end{array}\right]=\left[\begin{array}{cc}
A & 0 \\
0 & A_{Q}
\end{array}\right]\left[\begin{array}{l}
\Delta x \\
e_{Q}
\end{array}\right]+\left[\begin{array}{l}
B \\
0
\end{array}\right] \Delta u=\bar{A}\left[\begin{array}{c}
\Delta x \\
e_{Q}
\end{array}\right]+\bar{B} \Delta u} \\
\Delta \tilde{y}=\left[\begin{array}{ll}
C & -C_{Q}
\end{array}\right]\left[\begin{array}{l}
\Delta x \\
e_{Q}
\end{array}\right]=\bar{C}\left[\begin{array}{l}
\Delta x \\
e_{Q}
\end{array}\right]
\end{array}\right.
$$

Now we have the following:

Lemma: $\left(\bar{A}=\left[\begin{array}{cc}A & 0 \\ 0 & A_{Q}\end{array}\right], \bar{B}=\left[\begin{array}{l}B \\ 0\end{array}\right], \bar{C}=\left[\begin{array}{ll}C & -C_{Q}\end{array}\right]\right)$ ASPR if and only if $(A, B, C)$ ASPR.

Proof: $\bar{C} \bar{B}=\left[\begin{array}{ll}C & -C_{Q}\end{array}\right]\left[\begin{array}{l}B \\ 0\end{array}\right]=C B>0$ and 
$\bar{P}(s) \equiv \bar{C}(s I-\bar{A})^{-1} \bar{B}=\left[\begin{array}{ll}C & -C_{Q}\end{array}\right]\left[\begin{array}{cc}(s I-A)^{-1} & 0 \\ 0 & \left(s I-A_{Q}\right)^{-1}\end{array}\right]\left[\begin{array}{l}B \\ 0\end{array}\right]=C(s I-A)^{-1} B=P(s)$ is minimum phase. \#

So there exists $G_{e}^{*}$ such that $\left(\bar{A}_{C} \equiv \bar{A}+\bar{B} G_{e}^{*} \bar{C}, \bar{B}, \bar{C}\right)$ is Strictly Positive Real (SPR) when $(A, B, C)$ is ASPR. Consequently, as is well known from the Kalman-Yacubovic Theorem:

$$
\exists \bar{P}, \bar{Q}>0 \ni\left\{\begin{array}{l}
\bar{A}_{C}^{T} \bar{P}+\bar{P} \bar{A}_{C}=-\bar{Q} \\
\bar{P} \bar{B}=\bar{C}^{T}
\end{array}\right.
$$

We modify the adaptive control law with the RMF:

$$
\left\{\begin{array}{l}
u_{p} \equiv G_{e} \tilde{e}_{y}+G_{D} \phi_{D} \\
\tilde{e}_{y} \equiv y_{p}-\hat{y}_{Q} \\
\dot{\hat{x}}_{Q}=A_{Q} \hat{x}_{Q}+B_{Q} u_{p} \\
\hat{y}_{Q}=C_{Q} \hat{x}_{Q}
\end{array}\right.
$$

with modified adaptive gains given by:

$$
\left\{\begin{array}{l}
\dot{G}_{e}=-\tilde{e}_{y} \tilde{e}_{y}^{T} h_{e} ; h_{e}>0 \\
\dot{G}_{D}=-\tilde{e}_{y} \phi_{D}^{T} h_{D} ; h_{D}>0
\end{array}\right.
$$

Finally, we have the following stability result:

Theorem: In Eq. (9), let $(A, B, C)$ ASPR, $A_{Q}$ stable, $\phi_{D}$ bounded. Then the Modified Adaptive Controller with

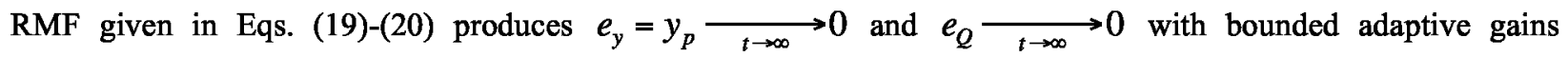
$\left(G_{e}, G_{D}\right)$.

Proof: From Eq. (19), we use $u_{p} \equiv G_{e} \tilde{e}_{y}+G_{D} \phi_{D}$ to obtain:

$$
\begin{aligned}
\Delta u & \equiv u_{p}-u_{*} \\
& =\left[G_{e} \tilde{e}_{y}+G_{D} \phi_{D}\right]-\left[S_{2}^{*} L\right] \phi_{D} \\
& =G_{e}^{*} \tilde{e}_{y}+\Delta G \eta
\end{aligned}
$$

where $\left\{\begin{array}{l}\Delta G_{e} \equiv G_{e}-G_{e}^{*} \\ \Delta G_{D} \equiv G_{D}-\left(S_{2}^{*} L\right) \\ \Delta G \equiv G-G_{*}=\left[\begin{array}{ll}\Delta G_{e} & \Delta G_{D}\end{array}\right] \\ \eta \equiv\left[\begin{array}{l}\tilde{e}_{y} \\ \phi_{D}\end{array}\right]\end{array}\right.$

Then we obtain:

$$
\left\{\begin{array}{l}
\dot{\zeta}=\bar{A} \zeta+\bar{B} \Delta u=\bar{A}_{C} \zeta+\bar{B} w \\
\tilde{e}_{y}=\bar{C} \zeta
\end{array}\right.
$$


with $\zeta \equiv\left[\begin{array}{l}\Delta x \\ e_{Q}\end{array}\right], w \equiv \Delta G \eta$, and $\overline{A_{C}} \equiv \bar{A}+\bar{B} G_{e}^{*} \bar{C}$.

From Eq. (20) we can see that:

$$
\dot{G}=\Delta \dot{G}=-\tilde{e}_{y} \eta^{T} h ; \quad h \equiv\left[\begin{array}{cc}
h_{e} & 0 \\
0 & h_{D}
\end{array}\right]>0
$$

Since $(A, B, C)$ is ASPR, and by the lemma, so is $(\bar{A}, \bar{B}, \bar{C})$, we can define $V(\zeta) \equiv \frac{1}{2} \zeta^{T} \bar{P} \zeta$ with $\bar{P}, \bar{Q}>0$ as in Eq. (18). Then $\dot{V}(\xi) \equiv-\frac{1}{2} \xi^{T} \bar{Q} \zeta+\left\langle\tilde{e}_{y}, w\right\rangle$. Define $V(\Delta G) \equiv \frac{1}{2} \operatorname{tr}\left(\Delta G h^{-1} \Delta G^{T}\right) \Rightarrow \dot{V}(\Delta G)=-\left\langle\tilde{e}_{y}, w\right\rangle$ using Eq. (23). Then $V \equiv V(\zeta)+V(\Delta G) \Rightarrow \dot{V}=-\frac{1}{2} \xi^{T} \bar{Q} \zeta=-W(\zeta) \leq 0$. So, we have $(\zeta, \Delta G)$ bounded and, from Barbalat's Lemma used on $W(\zeta), \zeta \equiv\left[\begin{array}{l}\Delta x \\ e_{Q}\end{array}\right] \underset{t \rightarrow \infty}{\longrightarrow} 0$ which leads to $e_{y} \equiv y_{p}=y_{p}-y_{*}=C \Delta x \underset{t \rightarrow \infty}{\longrightarrow} 0$ and $e_{Q} \longrightarrow t_{t \rightarrow \infty} 0$ with $G=G_{*}+\Delta G$ bounded, as desired. \#

\section{Simulation}

In this section, we investigate the apparent coupling between the drive train torsional mode and the blade flap mode in the FAST simulation and take appropriate corrective action in the controllers. We start with the baseline PI and the adaptive collective pitch controllers described in Section IV. These controllers are augmented with a Residual Mode Filter designed for the drive train torsional mode. We obtained linear models of the FAST states at trim with a constant wind inflow speed of $16 \mathrm{~m} / \mathrm{s}$ and a blade pitch angle of 0.133 radians. The disturbance from the drive train torsional mode was observed at these operating conditions. The linearized state space matrices were then averaged around the rotor disk. The resulting matrices were put into real diagonal form to identify the components corresponding to the drive train torsional mode. The modal representation of the drive train torsional mode represents the $Q$-modes, or troublesome modes, of the plant. The transfer function of the RMF for the drive train torsional mode is given by:

$$
T(s)=\frac{791.1534 s-38.3545}{s^{2}+0.0121 s+430.2993}
$$

The gains for the adaptive controller with the augmented RMF were $h_{e}=4.0$ and $h_{D}=0.3$. The results of adding this filter to the baseline PI and the adaptive controllers can be seen in Fig. 5. In both cases, the RMF improved the generator speed tracking of the controller.

\section{Conclusion}

A new control approach has been proposed to reduce the interactions between structural modes of a wind turbine during Region 3 operation. We developed the theory for disturbance accommodating adaptive control with a residual mode filter. The RMF is used to filter out troublesome modes from the plant that might inhibit the adaptive controller. This approach has the advantage over a low-pass filter or notch filter of exactly cancelling only the known troublesome modes. We used this approach to design a controller to regulate generator speed, reject step disturbances of unknown amplitude, and accommodate structural modes for the FAST simulator. This new adaptive control method shows promise for improved generator speed regulation in Region 3 for flexible turbines.

The trend in wind turbine design is toward larger, more flexible turbines with structural modes that can be easily excited by the turbulent operating environment. Wind turbine manufacturers are highly motivated to reduce component fatigue and failure. The new control approach described in this paper could help mitigate the fatigue and failure caused by excitation of structural modes. 


\section{References}

${ }^{1}$ Fingersh, LJ, Johnson, KE. Baseline results and future plans for the NREL Controls Advance Research Turbine. Proceedings of the $23^{\text {rd }}$ AIAA Aerospace Sciences Meeting and Exhibit Wind Energy Symposium 2004; 87-93.

${ }^{2}$ Wright, AD, Stol, KA, Fingersh, LJ. Progress in implementing and testing state-space controls for the controls advanced research turbine. Proceedings of the 24 $4^{\text {th }}$ AIAA Aerospace Sciences Meeting and Exhibit Wind Energy Symposium 2005; 88-100.

${ }^{3}$ Narendra, KS, Annaswamy AM. Stable Adaptive Systems. Prentice-Hall: New Jersey, 1989.

${ }^{4}$ Åström, KJ, Wittenmark, B. Adaptive Control ( $2^{\text {nd }}$ edition). Addison-Wesley: Massachusetts, 1995.

${ }^{5}$ Wen, JT, Balas, MJ. Robust adaptive control in Hilbert space. Journal of Mathematical Analysis and Application 1989; 143(1): 1-26.

${ }^{6}$ Balas, MJ. Finite-dimensional direct adaptive control for discrete-time infinite-dimensional linear systems. Journal of Mathematical Analysis and Applications 1995; 196(1): 153-171.

${ }^{7}$ Fuentes, RJ, Balas, MJ. Direct adaptive rejection of persistent disturbances. Journal of Mathematical Analysis and Applications 2000; 251(1): 28-39.

${ }^{8}$ Frost, SA, Balas, MJ, and Wright, AD. Direct adaptive control of a utility-scale wind turbine for speed regulation, International Journal of Robust and Nonlinear Control, 2009, 19(1): 59-71, DOI: 10.1002/mc.1329.

${ }^{9}$ Freeman, JB, Balas, MJ. Direct model-reference adaptive control of variable speed horizontal-axis wind turbines. Wind Engineering 1998; 22: 209-218.

${ }^{10}$ Johnson, KE, Fingersh, LJ. Adaptive pitch control of variable-speed wind turbines. Collection of Technical Papers $-45^{\text {th }}$ AIAA Aerospace Sciences Meeting and Exhibit 2007; 18:12391-12398.

${ }^{11}$ Wright, AD, Balas, MJ, Fingersh, LJ. Testing state-space controls for the controls advanced research turbine. Transactions of the ASME. Journal of Solar Energy Engineering 2006; 128(4): 506-515.

${ }^{12}$ Wright, AD, Balas, MJ. Design of controls to attenuate loads in the controls advanced research turbine. Transactions of the ASME. Journal of Solar Energy Engineering 2004; 126(4): 1083-91.

${ }^{13}$ Jonkman, JM, Buhl, ML. FAST user's guide, National Renewable Energy Laboratory, NREL/EL-500-38230, Golden, Colorado, August, 2005.

${ }^{14}$ Johnson, C.D. Theory of disturbance-accommodating controllers. Control \& Dynamic Systems, Advances in Theory and Applications, Leondes, CT. ed. Academic Press: New York, 1976; 12: 387-489.

${ }^{15}$ Frost, SA, Balas, MJ, and Wright, AD. Adaptive Control of a Utility-Scale Wind Turbine Operating in Region 3, Proceedings of the $28^{\text {th }}$ AIAA Aerospace Sciences Meeting and Exhibit Wind Energy Symposium, Orlando 2009.

${ }^{16}$ National Renewable Energy Lab., NWTC Design Codes (FAST), http://wind.nrel.gov/designcodes/simulators/fast/, National Renewable Energy Lab., Golden, CO, May 26, 2008.

${ }^{17}$ Balas, MJ, Finite-dimensional controllers for linear distributed parameter systems: Exponential stability using Residual Mode Filters," J. Mathematical Analysis \& Applications, Vol. 133, pp. 283-296, 1988. 


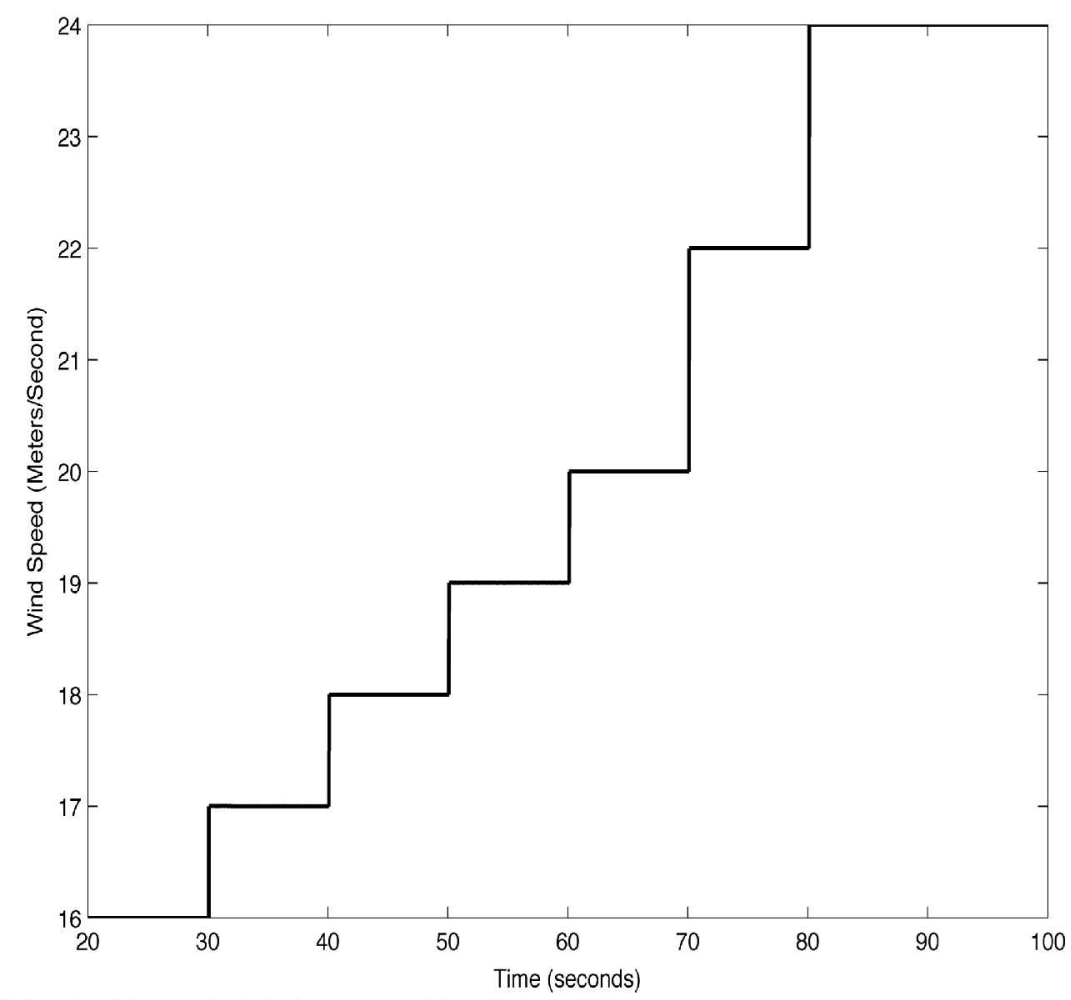

Fig. 1. Step wind inflow used in simulations.

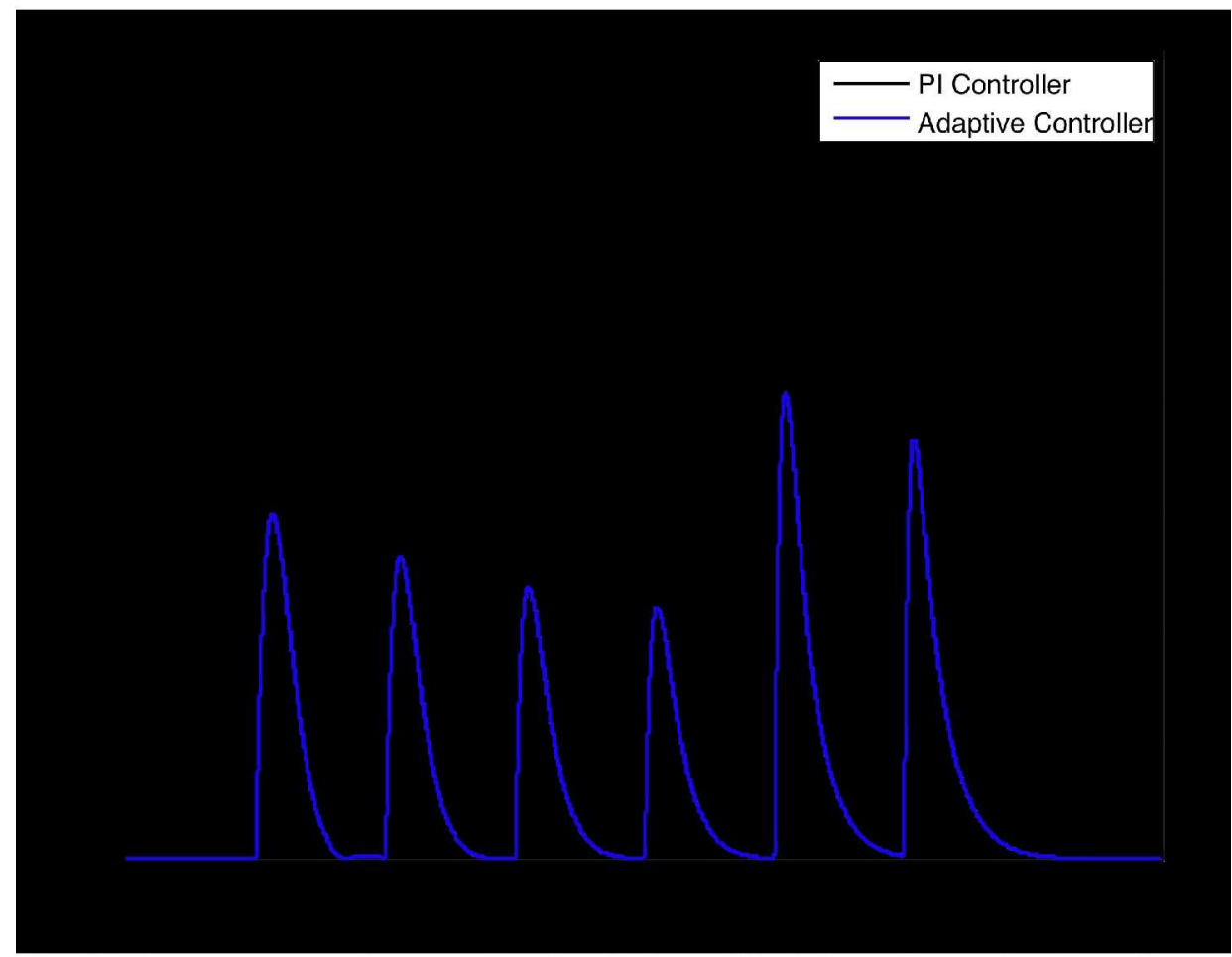

Fig. 2. Generator speed errors for simulation with drivetrain torsional mode and first flapwise blade mode DOFs disabled for baseline PI controller and adaptive controller. 


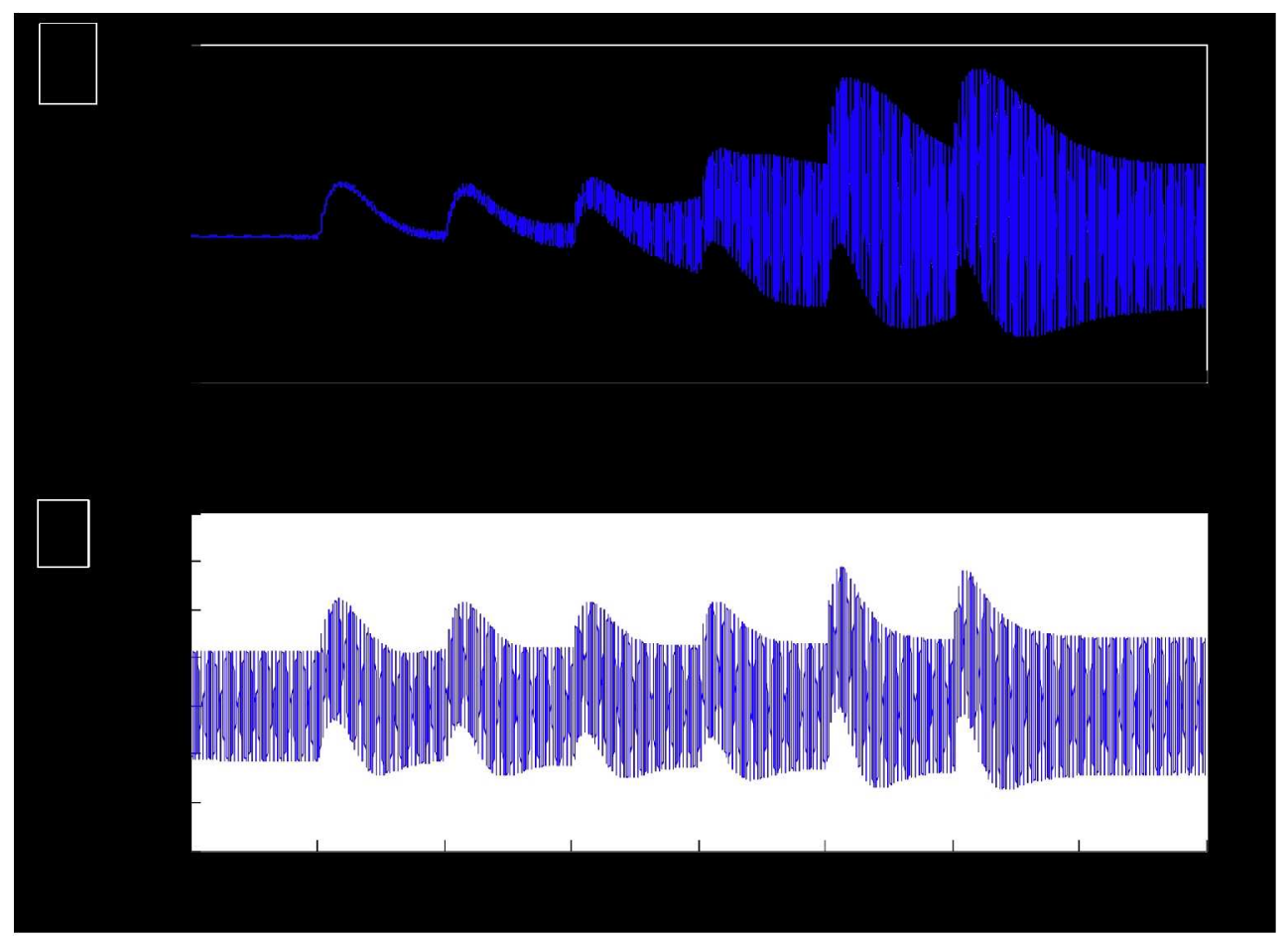

Fig. 3. Generator speed for simulation with drivetrain torsional and first flapwise blade mode DOFs enabled for (a) baseline PI controller and (b) adaptive controller.

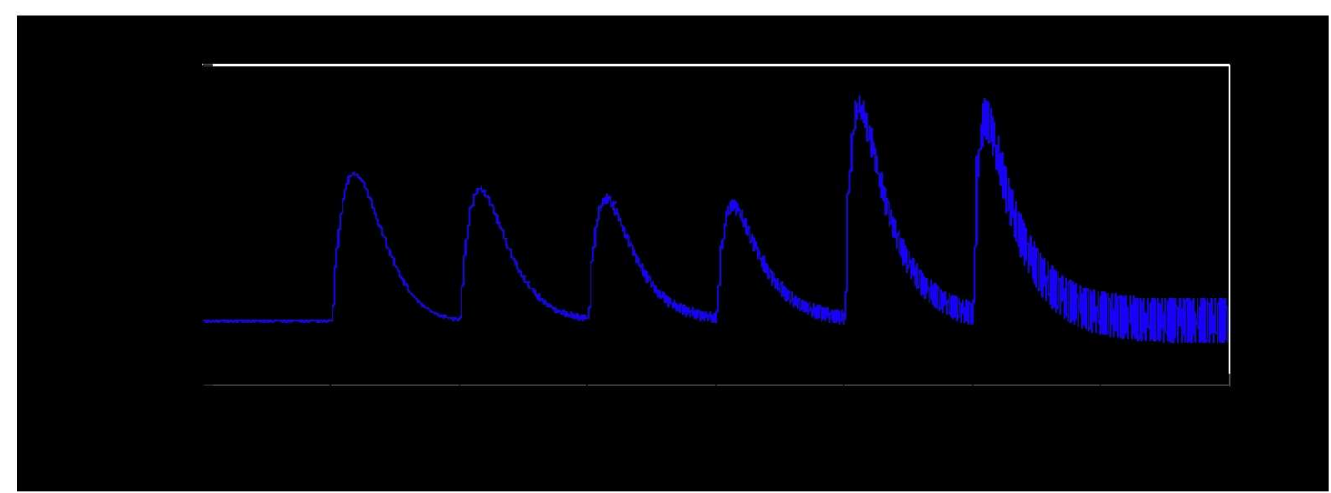

Fig. 4. Generator speed for simulation with drivetrain torsional and first flapwise blade DOFs enabled for baseline PI controller with low-pass filter of generator speed. 


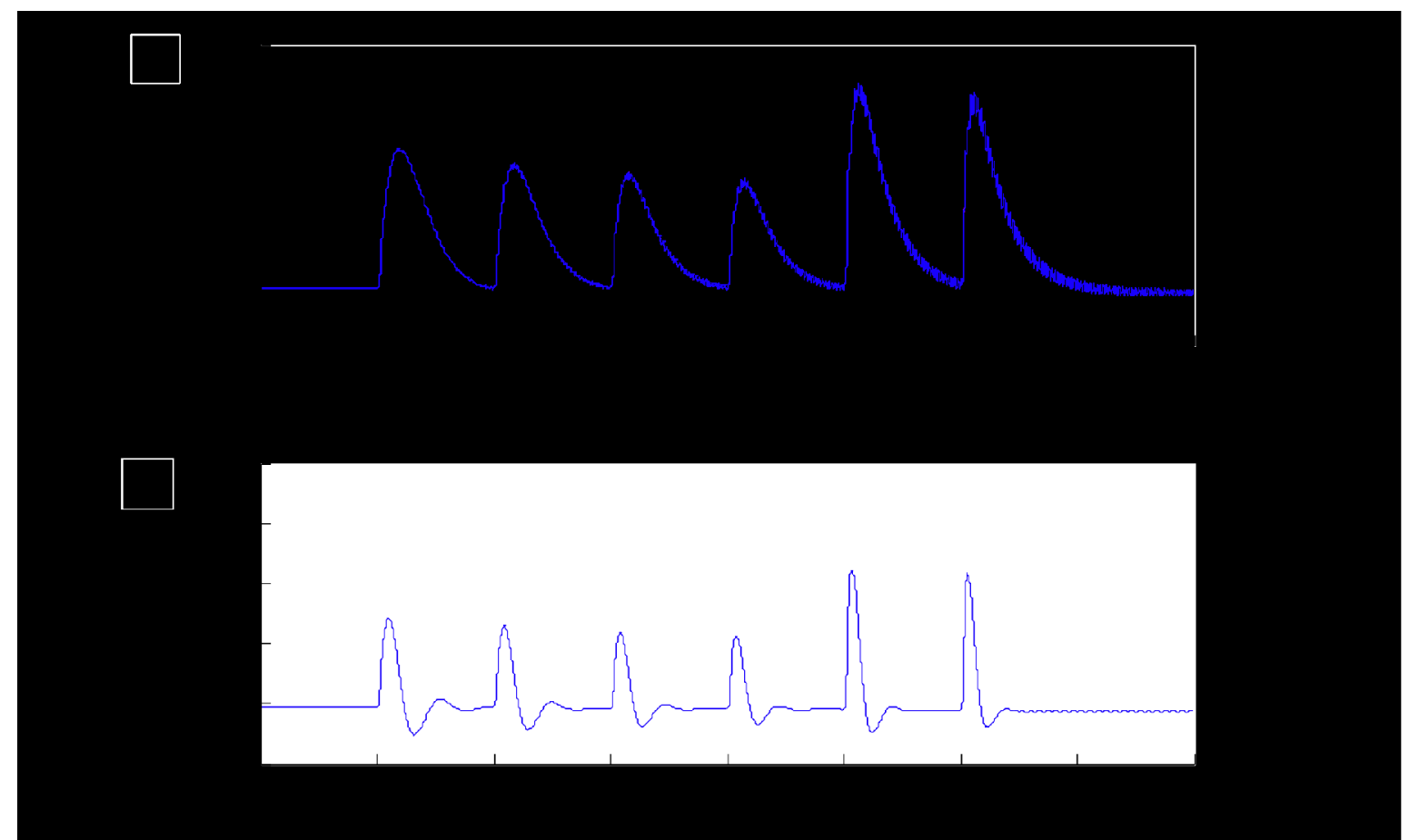

Fig. 5. Generator speed for simulation with drivetrain torsional and first flapwise blade mode DOFs enabled for (a) baseline PI controller with RMF and (b) adaptive controller with RMF. RMF was designed for drivetrain torsional mode. 